\title{
Validating the Applicability of Agile Requirement Generation Model
}

\author{
Mani Sahore \\ Lovely Professional University \\ Phagwara (Punjab)
}

\author{
Aseem Kumar \\ Assistant professor \\ Lovely Professional University \\ Phagwara (Punjab)
}

\begin{abstract}
We all know that agile methodology for software development is an alternative to waterfall methodology for software development. In today's scenario, agile methods have caught the attention of software engineers worldwide. The reasons of this popularity are its iterative nature, quick delivery of software, face to face communication, less need of lengthy documentation etc. Agile methods focus on accommodating change even late in the development lifecycle. Though agile method are successfully applied on small softwares (whose development periods are less than 1-2 years), the main issue comes when someone want to apply agile methodology on large systems (development period greater than 2 years). One of the most important issues in the development of larger scale complex systems is accommodating changes to requirements. Someone proposed a soft-structured framework which combines the principles of agile and conventional software development that solves the issue of rapidly changing requirements for larger scale systems. The framework consists of two parts. First one is a soft structured requirements gathering approach that reflects the agile philosophy i.e. the Agile Requirements Generation Model and second one is a tailored development process that can be applied to either small or larger scale systems. The main work is to validate the applicability of the Agile RGM. So this paper validates the applicability of agile requirement generation model with the help of a project.
\end{abstract}

Keywords: Agile Software Development; Agile requirement generation model; Use cases; UML;

\section{Introduction}

Agile processes have grown increasingly popular. Changes in requirements are welcomed at every stage of the software development. There are some important benefits of agile. Due to these benefits the number of organisations is adopting agile practices. Improved quality, Greater return on investment, shorter time to market, Enhanced customer relationships, Better team morale [1]. This paper provides an overview of agile requirement generation model, methodology how to validate the Requirement Generation model, analysis of Agile RGM with the help of project, some observations, precaution steps which are concluded after statistical analysis .In addition, the paper also focuses on the limitations of agile requirement generation model. However the significance of this paper is to validate the applicability of agile requirement generation model to its users in a very efficient way.

\section{Agile Requirement Generation Model}

The main work of Agile Requirements Generation Model (RGM) is to accommodate change. The Requirements Generation Model provides the necessary structure for the Agile RE process, and thereby helps guide the practitioners. According to Agile RGM, if a new feature is recognised at a later stage then we have to first break that feature into story and arrange that stories according to their priorities and then break down the highest business value story or highest number of priority into number of tasks and then implement the task in the development phase.Objective is to validate the applicability of requirement generation model on large sized projects. The results after the validation of RGM will depict whether it is applicable for large systems or not. If yes then this will be very beneficial for the large organizations. Diagrammatic representation of agile requirement generation model is as given below: 


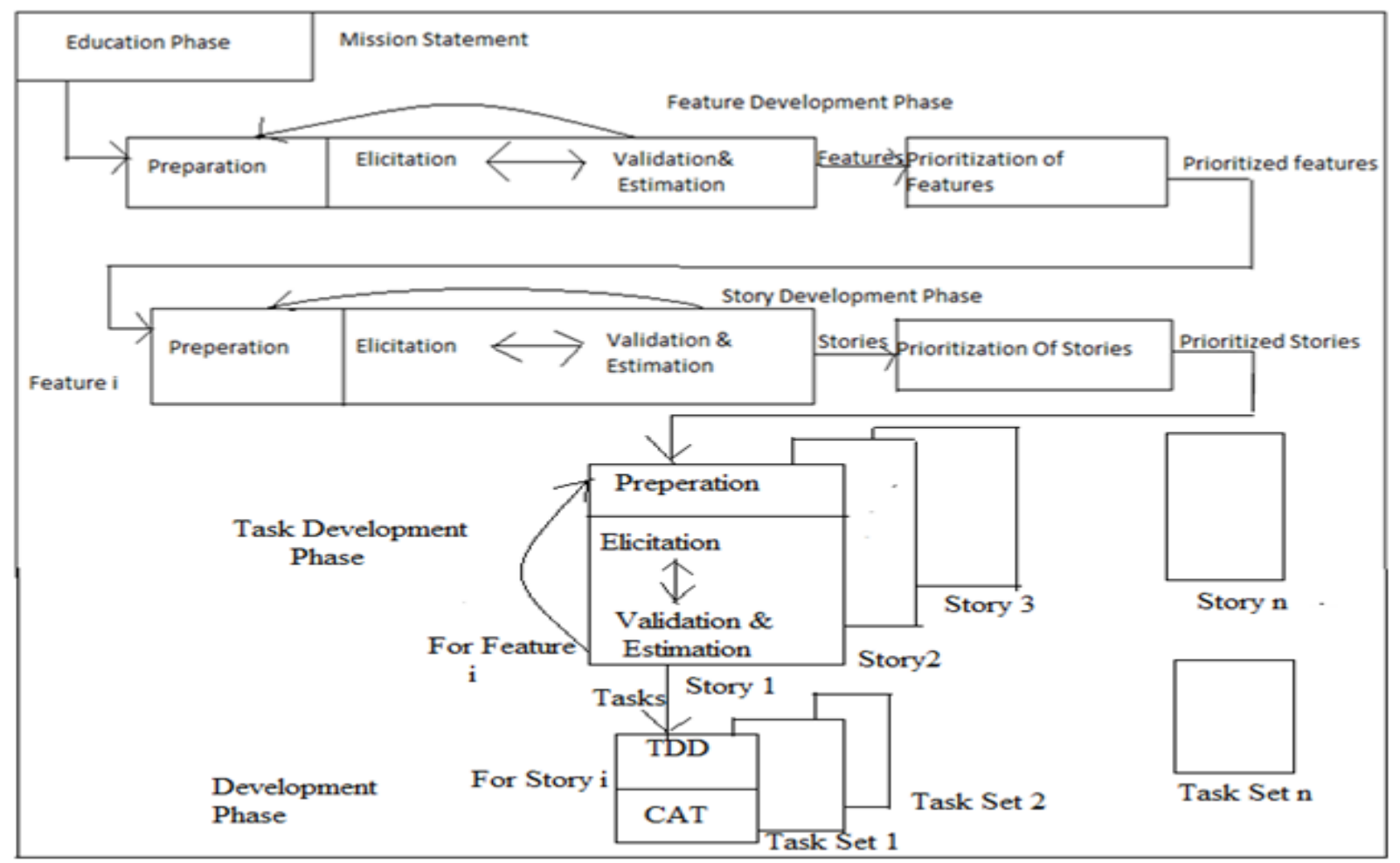

Fig 1 : Agile Requirement Generation Model

\section{Methodology}

Methodology consists of the steps in a sequence which we have to follow while solving a particular type of problem. It consists of specific components such as phases, tasks, methods, techniques and tools. Methodology should be correct and in a proper sequence.[2]

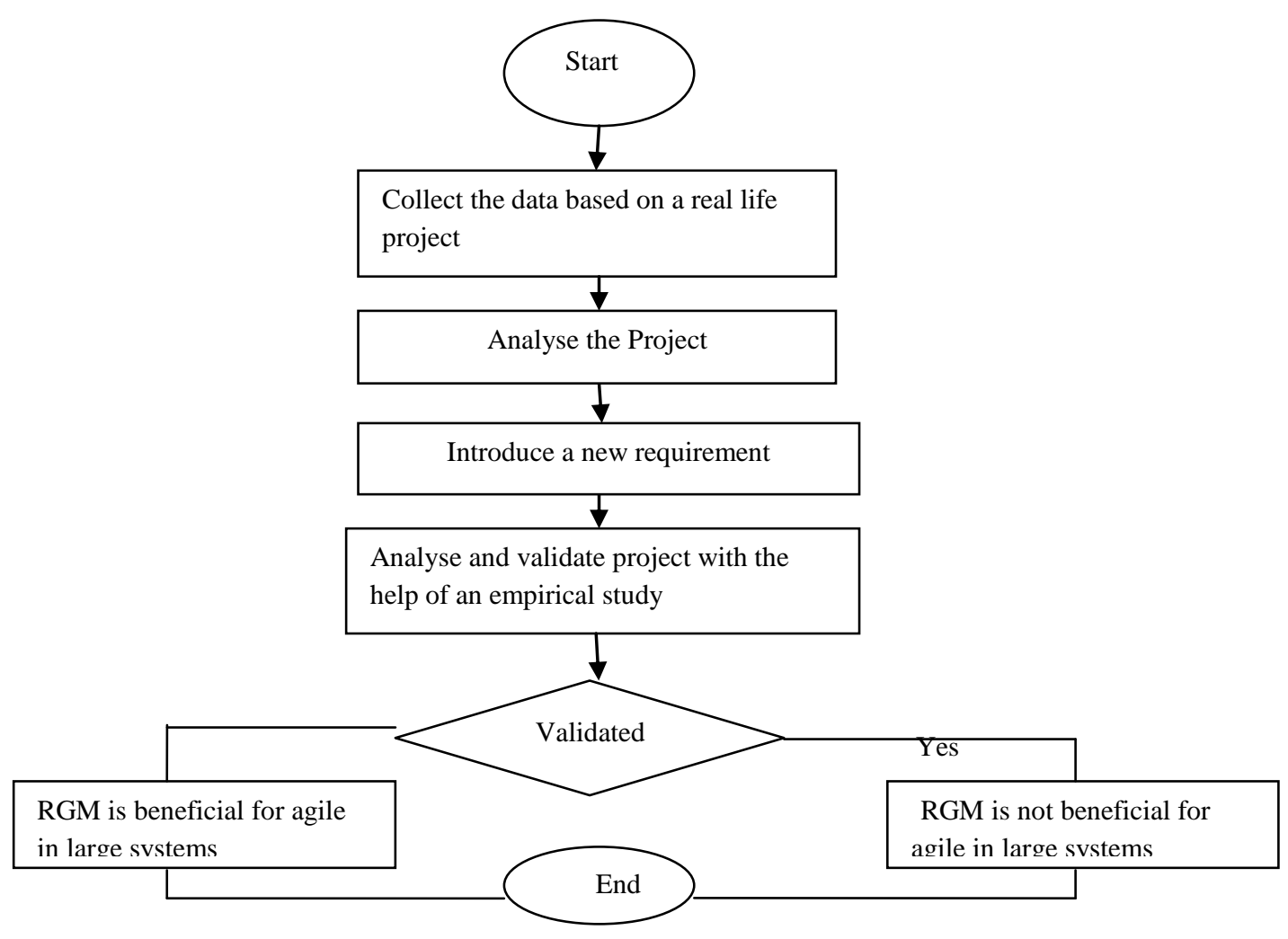

Fig 2: Methodology 


\section{Results}

In the results part, first I want to implement methodology with the help of my project which i have taken for the statistical analysis. I have given general methodology above and now I am going to implement it.

\section{Implementation of the methodology}

\section{Step 1: Collect the data based on a project}

Starting from the scratch, the real life project which I have selected for the validation of Agile RGM is " Human Resource Management" made by trainers in ESoft technologies. The main work which I have to do is to validate the agile requirement generation model with the help of a project and show the developers of that project that if they want to adopt agile methodology then how that thing become possible. The graphical description that shows how agile requirement generation model can be validated with the help of a project can be shown through use cases, class diagrams.Tool used for data collection and analysis is Magic Draw UML.

\section{Step 2.Analyse the whole project in depth} Human Resource Management is a project which commonly deals with the online recruitment of fresher's. This project is a medium sized project and contains modules like Career module for Fresher's, online test Module, Recruitment Module, Training Module, Reimbursement Module Attendance Module, Payment Module.Before going to the implementation part, I want to discuss first the whole project, its modules in depth, how all module are interconnected to each other. As i already told that graphical description is shown with the help of UML. Various modules and their dependencies with one another is also shown with the help of Magic draw UML symbols like association, dependency, aggregation.

\section{Step 3: Introduce a new requirement}

After going into the depth of the whole project, there is a need of two more modules which are not there in the existing project. I feel that if these two facilities are added into the existing project, this will prove to be very beneficial for the employees. Two facilities are Accommodation facility and Transport facility. Now the main question arises how to implement requirements at a later stage. Let's discuss the working of Agile RGM and see how requirements are added with the help of agile RGM. If requirements are added at later stage then Agile RGM gets validated.

\section{Step 4: Implement the requirements with the help of agile RGM}

a)First requirement is accommodation facility. It will provide living facility to all the employees who got selected in the company. This facility will provide hostels to the employees, Paying Guest facility etc. This facility is not in the existing project so I am suggesting the developers of the project that if they add these requirements then this will be very beneficial to all the employees.

b)Second requirement is Transport facility will provide vehicles to the employees so that they can go from their living area to the company. Transport module will provide some facilities like employees can view the transport facility, they can register for transport facility if they want to avail it and also do payment online for this facility.

Now this thing becomes very much clear that what are the requirements that are going to add in the project. Now the question arises that how to add new requirements with the help of Agile RGM. According to Agile RGM, if a new requirement is to be recognised at a later stage in the development process, then according to Agile RGM there is a need of breaking down that requirement into further parts so that implementation at lower level becomes easy. We have to be first break that requirement into stories and arrange that stories according to their priorities. Then further break down that story into tasks and again prioritize that stories based upon their business values and then implement that task with the help of development phase. 


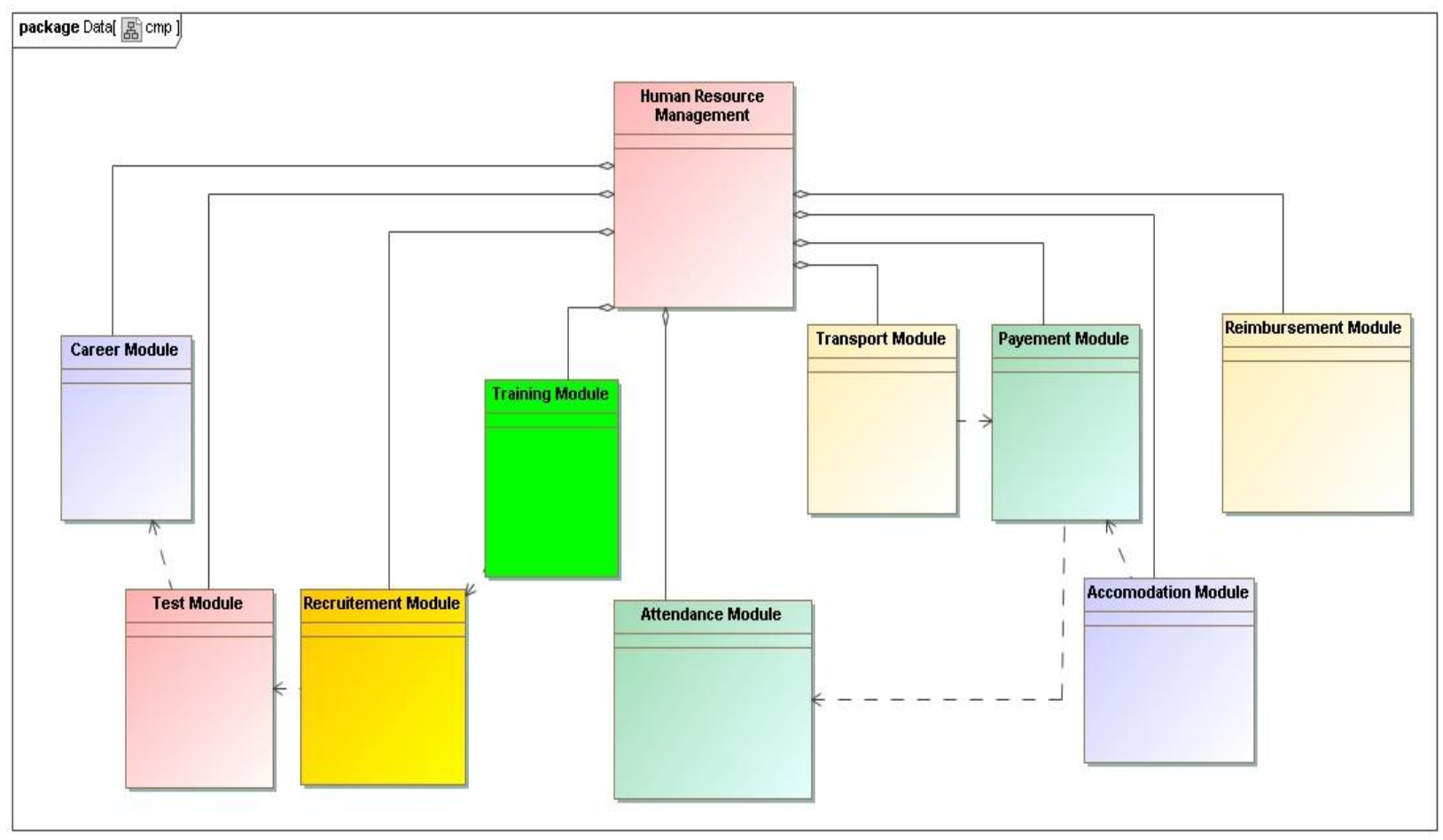

Fig 3 : Human Resource Management

\section{How to add new requirements with the help of Agile RGM}

According to Agile RGM, if a new feature is recognised at a later stage then we have to first break that feature into story and arrange that stories according to their priorities and then break down the highest business value story or highest number of priority into number of tasks and then implement the task in the development phase. Working is as given below:

\subsection{Education Phase}

First of all lets discuss the first phase of agile RGM i.e. Education Phase. The main motive of this phase is to establish communication between various stakeholders. In this phase developers learn the business process of the customer's. Also in this phase a product concept statement is created which is also called high level mission statement.Product concept statement is a statement which will answer the following questions like

\section{a)Who are the products Users?}

Answer: Here the product is Human Resource Management Project. So the product users are the fresher candidates who are looking for a job, all the employees who got recruited.

\section{b)What will the product do?}

Answer: Human resource management is a product that is providing online recruitment of fresher candidates, maintain the details of the employees like details regarding payment, attendance, details regarding reimbursement etc.

\section{c)What type of problems will the product solve?}

Answer: The basic problem lies with the fresher candidates are that they have to go to far places for their interviews. Also if they got selected then there is neither accommodation facility for them nor the transport facilities. So this product and the requirements which I am suggesting for addition will solve all the problems discussed above.

\subsubsection{Use case Diagram for Education Phase}

Use case describes the operations between developer and customer. 


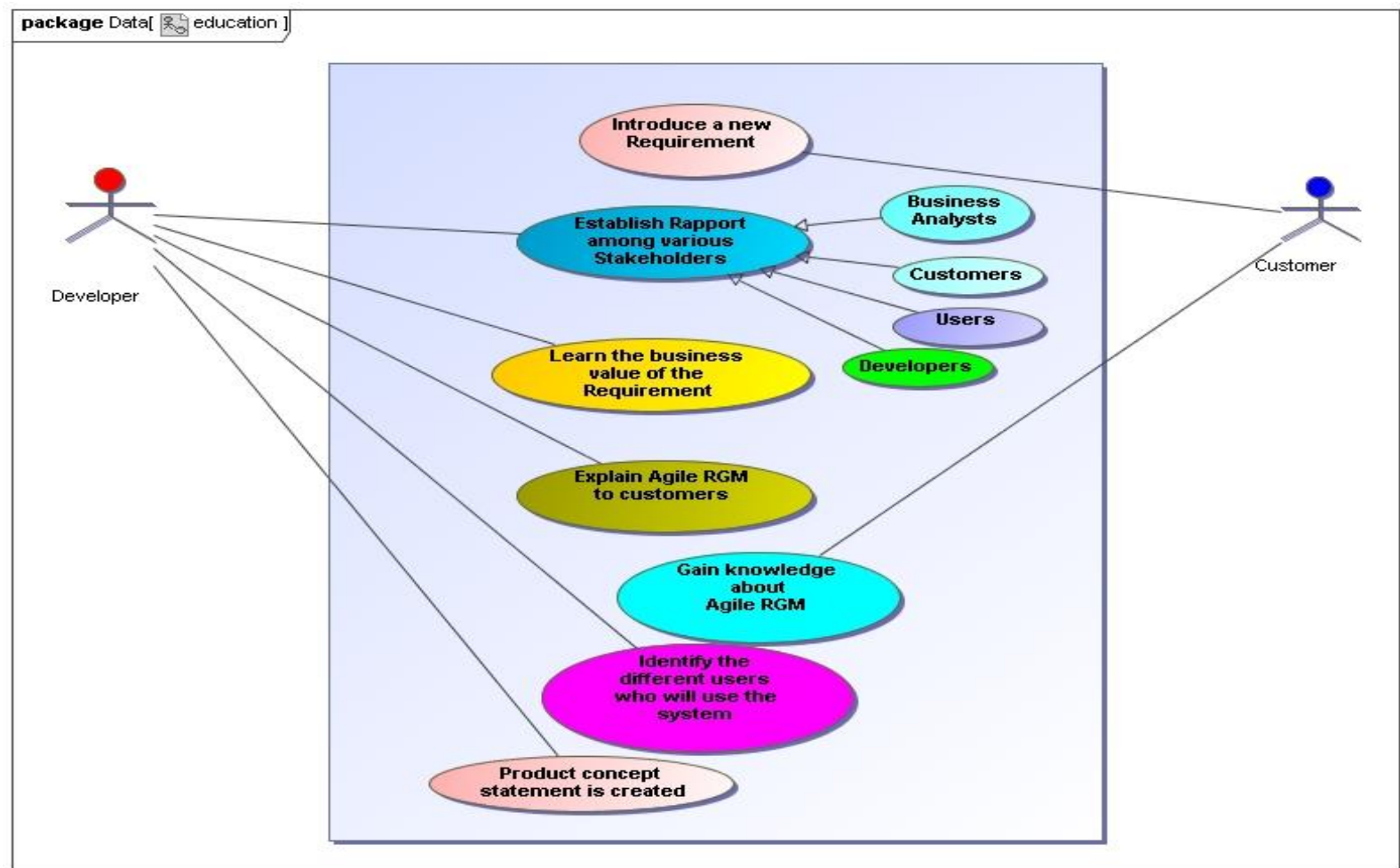

Fig 5: Use case diagram for Education Phase

\subsection{Feature Development Phase}

High level mission statement which is created in the above phase becomes the input of this phase. The features are extracted with the help of meetings from the product concept statement.

Table 1: Example for features

Accommodation and Transport is a feature that can be beneficial for E-Soft Technologies.

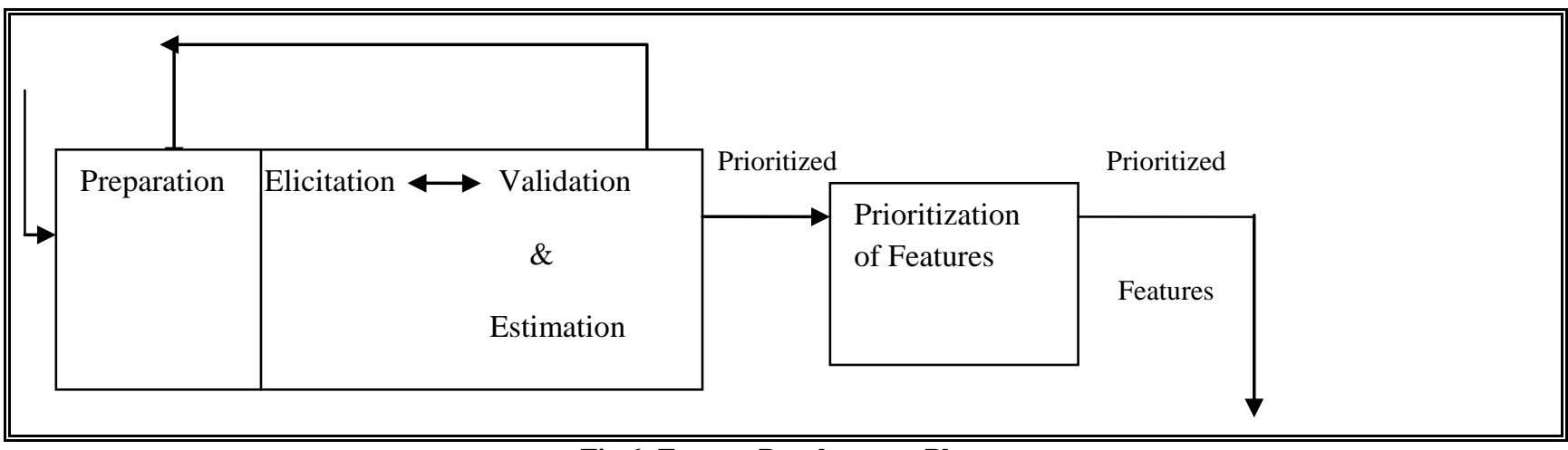

Fig 6: Feature Development Phase

\subsubsection{Preparation}

This activity is to arrange for a meeting among all the stakeholders to identify the major features of the system to be built.

\subsubsection{Elicitation}

How to gather requirements basically comes under this point. Brainstorming sessions involving all the stakeholders are generally an effective way to identify features. Open-ended interviews and focus group with the customers and users are other techniques to elicit features.

\subsubsection{Validation and Estimation}

The elicited features are validated and the time required to complete each feature is estimated.

\subsubsection{Prioritization}

Customers prioritize the identified set of features based on their needs and return on Investment. 
The output of the feature development phase is a prioritized feature stack. Hence, the prioritized feature stack is a collection of index cards containing features and sorted by their priorities.

\subsubsection{Use case for Feature Development phase for better understandability}

Use Case diagram for feature development phase depict the functionality of the whole phase. All the

communication activities between the customer and the developer can be easily shown with the help of use case diagrams.

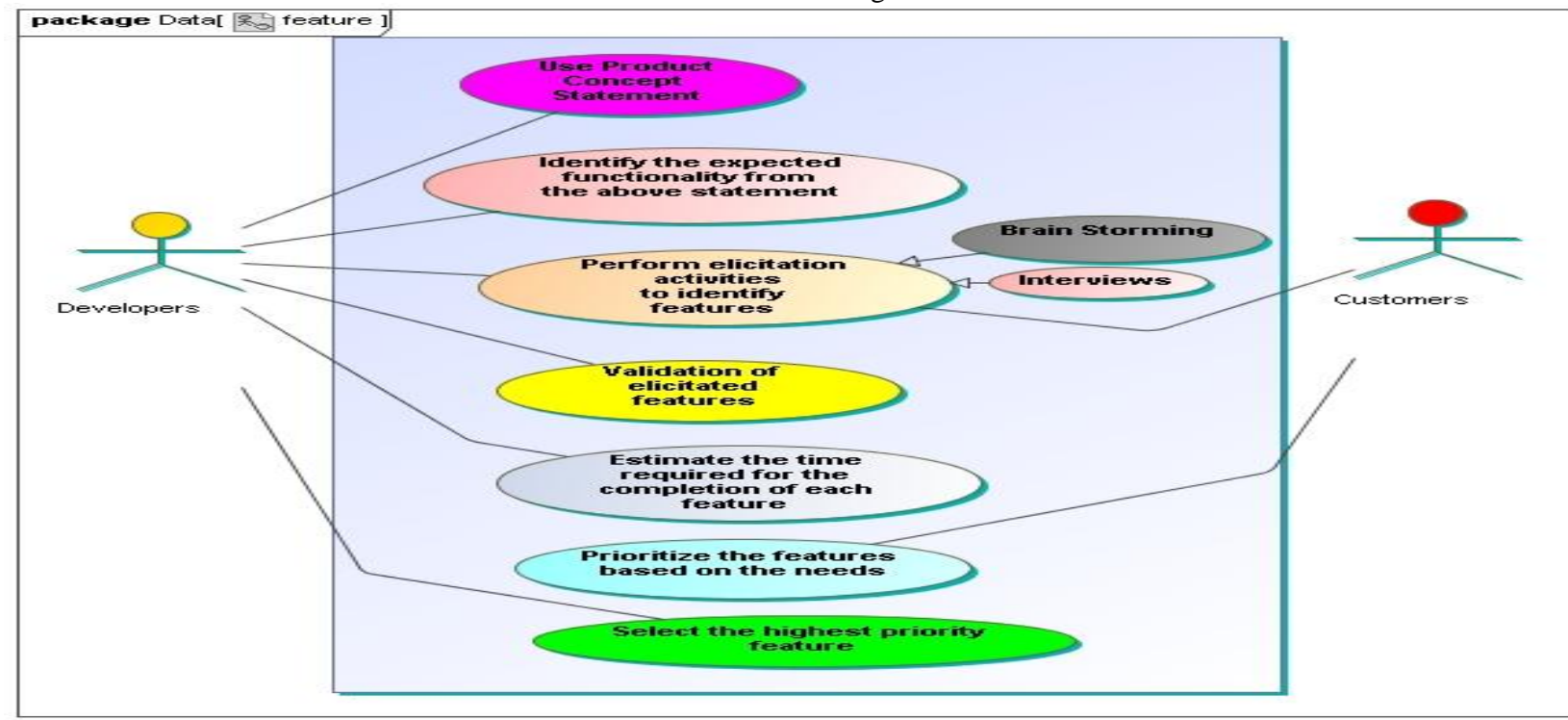

Fig 7: Use Case diagram for Feature Development Phase

\subsection{Story Development Phase}

Now there comes the further division of feature into stories. Explanation is same as in feature development phase.

Table 2: Example for Stories

Consider an Accommodation Facility for Fresher's Feature mentioned in table. The following are the three stories created for this feature.

1. He can avail the facility through credit card.

2. He can avail the facility direct from his salary.

3. He can use an e-check to make a payment and avail the facility.

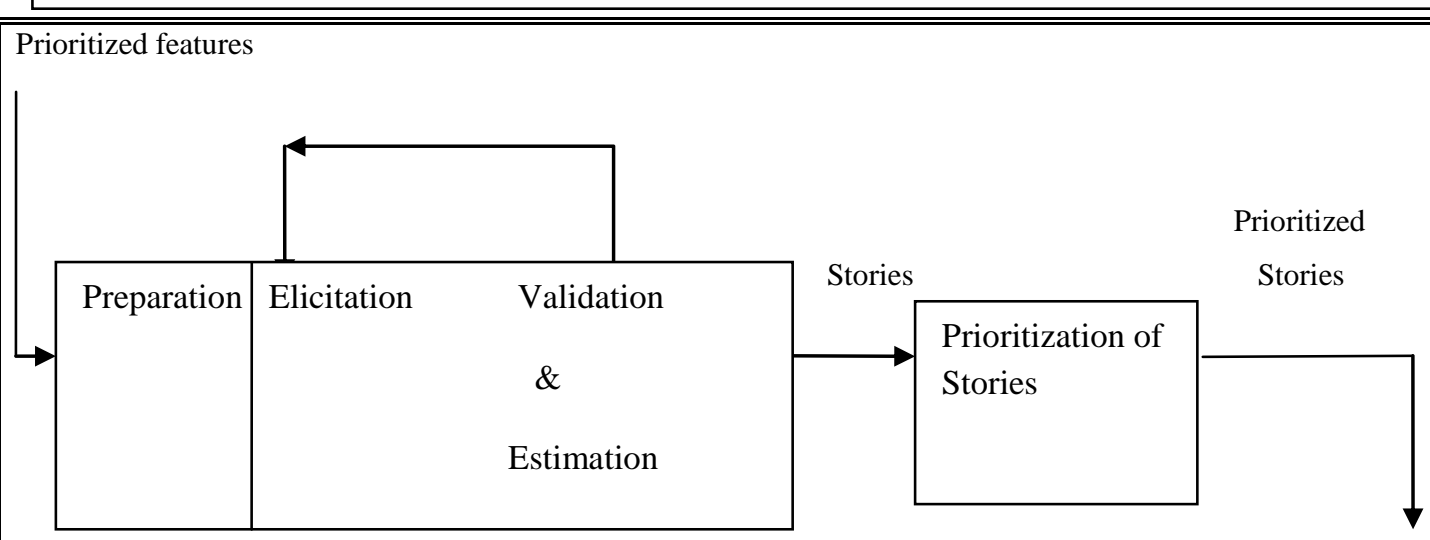

Fig 8: Story Development Phase 
The output of this phase is a Prioritized story stack. After a story or an initial set of stories are identified, tasks can be created and developed. Each story identified in the previous phase is decomposed into tasks by the development team.

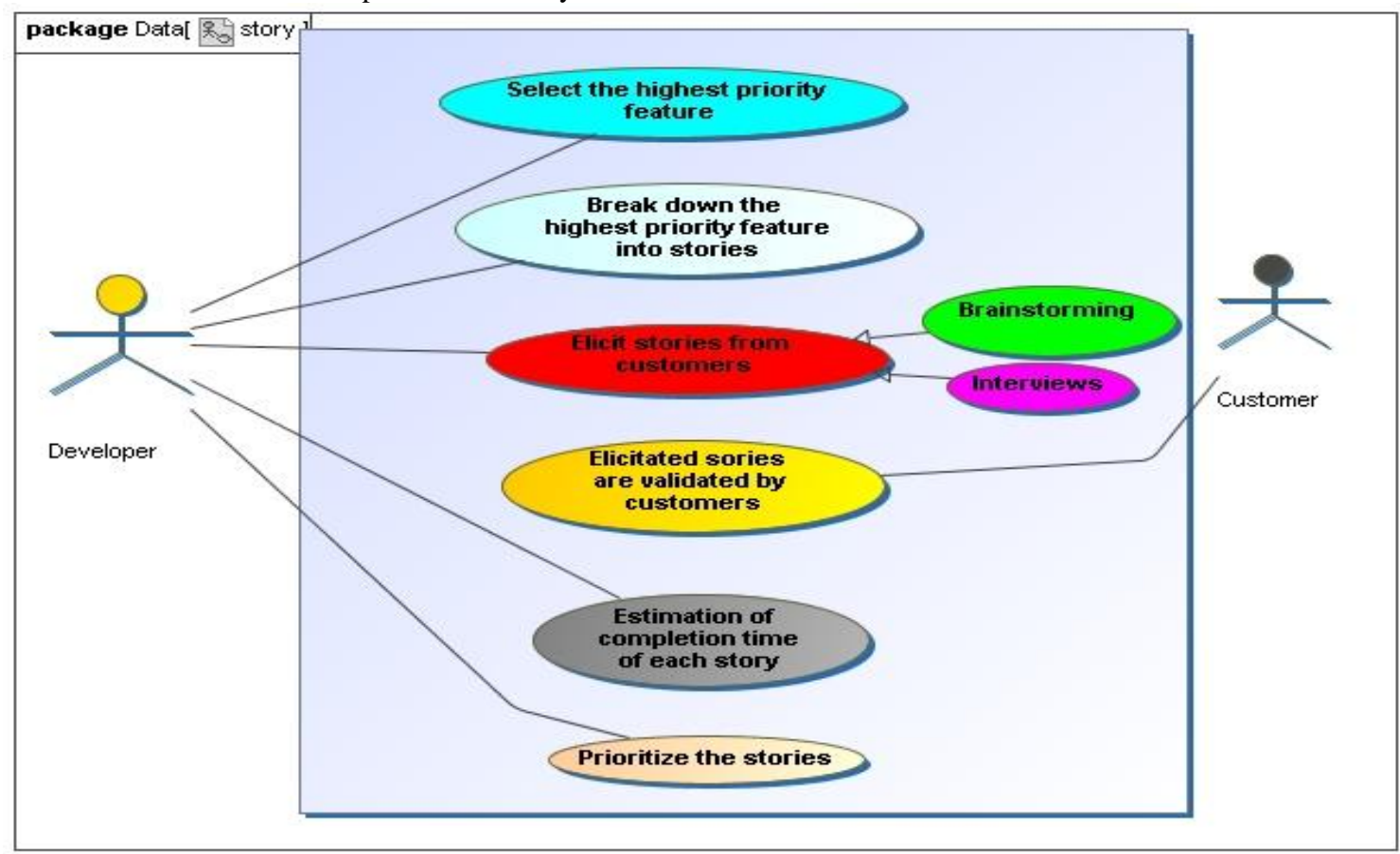

Fig 9: Use Case Diagram for Story development Phase

\subsection{Task Development Phase}

development Phase .

The stories chosen to be developed during the current iteration serve as the input to the Task

Table 3: Example for tasks

Example for tasks:

Consider the story "He can avail the accommodation facility through credit card." described in table 2. The following are the some of the tasks of this story.

1. Code credit card details page

2. Ensure that only Visa and Master cards are accepted 


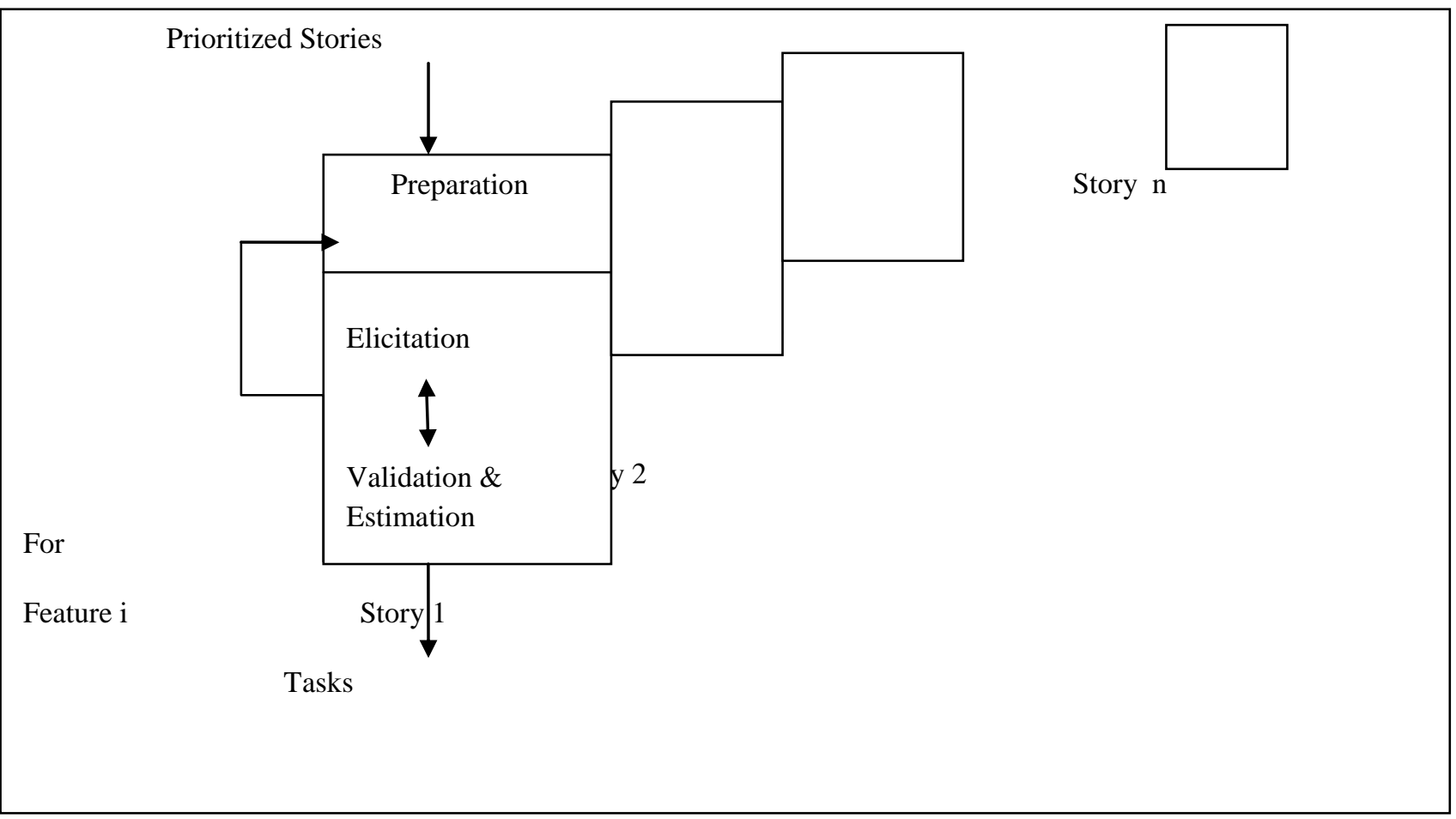

Fig 10: Task Development Phase

\subsubsection{Use Case Diagram for Task Development Phase}

Use case diagram for task development phase depicts the communication activities going on between customer and developer. How stories are broken down into number of tasks and how that tasks are prioritized all these things are very well shown in the use case diagram.

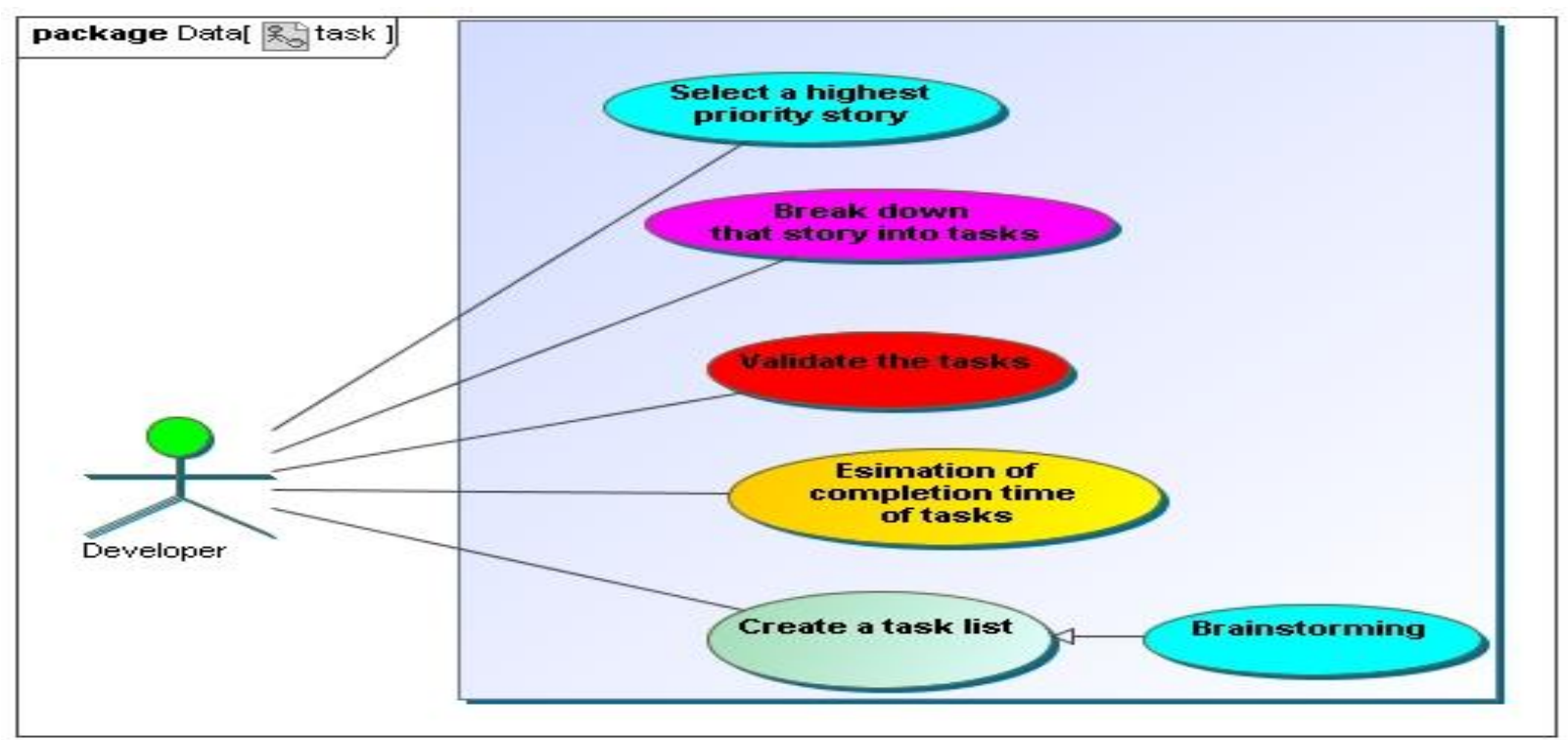

Fig 11: Use Case Diagram for Task Development Phase

\subsection{Development phase}

Development phase contains two things. Test driven development and customer acceptance tests.

\section{Test driven development}

It is a combination of Test First Development and refactoring. It is a development technique in which developers create unit test for task. This activity is 
done before the writing of code. Refactoring is basically refining of the code so that it can be better understood to its users. It is basically changing the design and structure of the code without changing the result. Refactoring basically eradicates dead code.

\section{Customer Acceptance tests}

This type of testing ensures that the resulting system meets the expectation of the customer. Basically the main disadvantage of acceptance testing is that they are very time consuming.[3]

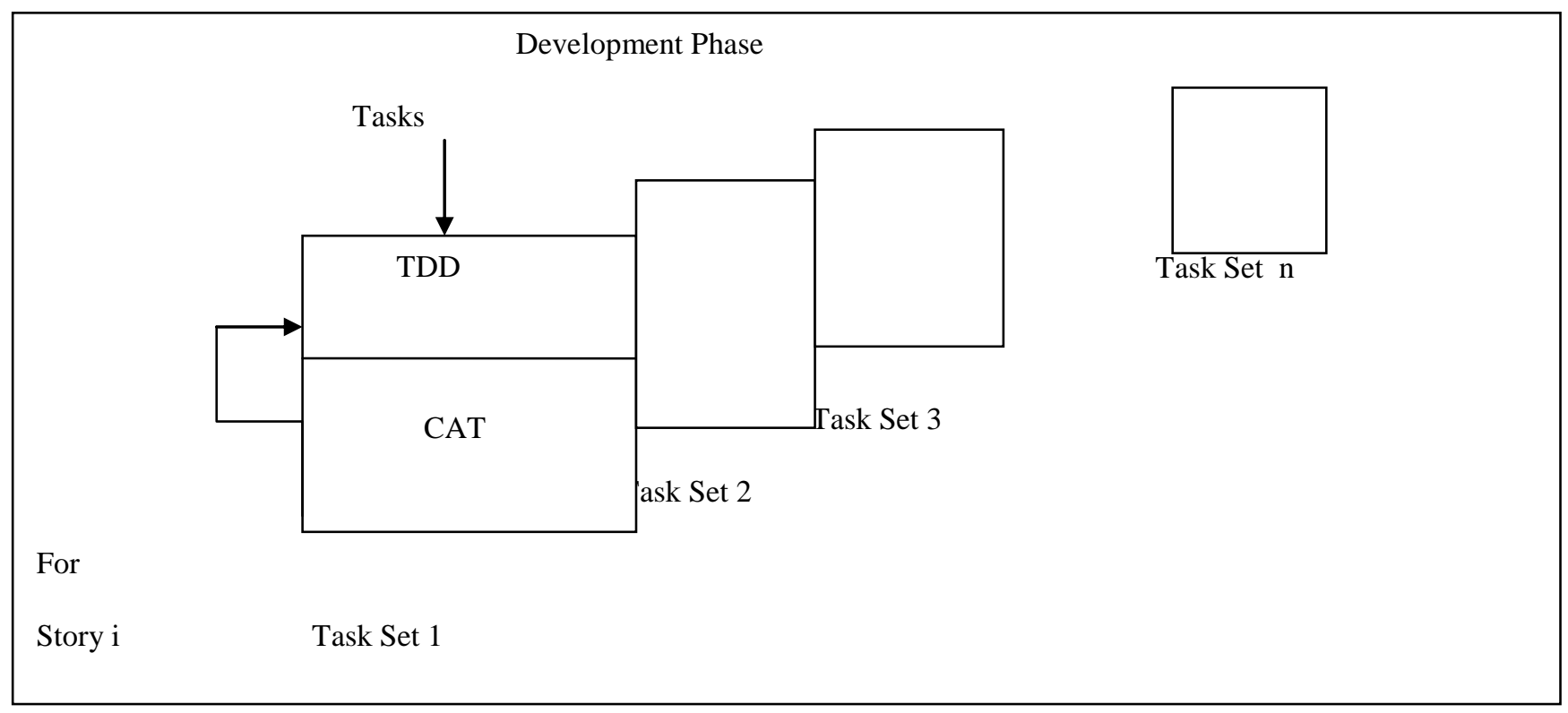

Fig 12 : Development Phase

5.5.3 Use case Diagram for Development development and customer acceptance testing. Phase

Use case diagram for development Phase depicts that how tasks are implemented with the help of task driven

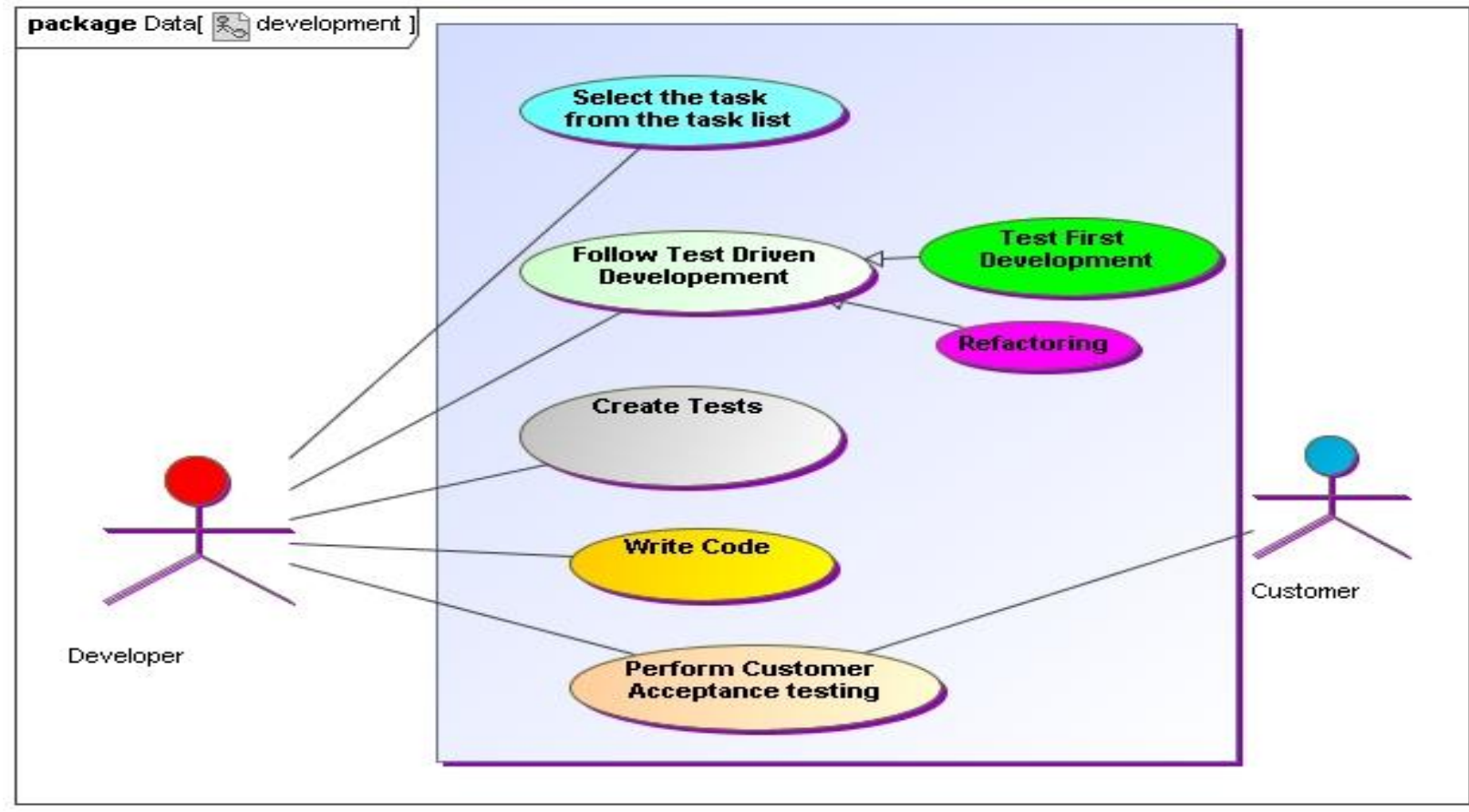

Fig 13: Use Case Diagram for Development Phase 


\section{Observations after Statistical Analysis}

Following discussions in the form of points are made after the results of the analysis.

\subsection{How this model accommodates change}

Change is possible due to the pre estimation of the completion of the features which are identified late in the development cycle. With pre-estimation, priority is also assigned to the features based on their benefits to the users and then features will be added to the prioritized feature stack. On identifying new features, existing priorities should be reassessed. For example I have introduced two features named transport facility and accommodation facility. It is obvious that when an employee joined the company and if his hometown is far from the company then he may need accommodation facility first and then transport facility. So accommodation facility has a higher priority than transport facility. However this result came only for small module change. I have introduced only the one module. In the later stages validity of the module will be checked by introducing large number of modules at later stages.

\subsection{What are the precaution steps that have to be take care of}

There are mainly 2 precautions which must be taken while requirement gathering. First one is requirement gathering must be from the expert person in his domain so that all the necessary features must be included in the final project and second precaution is requirements must be clear, unambiguous and consistent.

\subsection{Disadvantages observed after analysis}

Following disadvantages are observed after analysis which is as explained below:

a)If the pre-estimation time of late identified feature is longer than the time frame for a release cycle then the developers discard that feature with a motive to accommodate the timeline for the project.

b)Another disadvantage which we all know about agile, which is actually a benefit also is direct customer involvement. Agile RGM basically emphasis on face to face interaction with all the stakeholders with a motive to extract needs from them, gather details in quick time and validate the output of each phase. Agile development methodology demands that customer must be onsite full time. But this is not possible always and due to lack of communication with customer, it may breakdown the progress for large projects.
c)Another disadvantage is that feature does not support concurrency (multiple features can't be decomposed at one time) because in this particular phase all the stakeholders should try to identify all the features in advance with a purpose to determine the scope of a system. Also there is no multiple team members involved in this phase. Other phases of agile RGM are concurrent and multiple team members are involved in it. Benefit of Concurrency is that it results in faster development of product.

\section{Conclusion}

About RGM I reached to a conclusion that Agile RGM is very well suitable for introducing requirement at a later stage due to its division of breaking down requirements into stories and then division of stories into tasks. As we can see from the above diagrams of breaking down of features into stories and then stories into tasks, implementation of tasks with the help of task driven development and customer acceptance testing is very easy.

\section{Future Scope}

According to me there are 2 things that can be implemented in future.

a)There is a great need of a tool that supports the Agile RGM approach. If such type of tool gets developed then it will be very helpful in keeping track of the user needs, features, stories and tasks.

b)There is a great need of validation of Agile RGM on large scale project. The project which I have taken for testing the Agile RGM is a small scale project.

\section{References}

[1] Abrahamsson, P., \& Warsta, J. (2003). New Directions on Agile Methods: A Comparative Analysis. 25 International Conference on Software Engineering.

[2] http://en.wikipedia.org/wiki/Methodology

[3] Soundararajan, S. , \& Arthur, J. D. (2009) . A SoftStructured Agile Framework for Larger Scale Systems Development. 16th Annual IEEE International Conference and Workshop on the Engineering of Computer Based Systems. 